\title{
Epstein-Barr Virus-Positive Diffuse Large B-Cell Lymphoma Involving the Colon in a Patient With Ulcerative Pancolitis and Polymyositis on Long-Term Methotrexate Therapy
}

\author{
Michael D. Chang ${ }^{\mathrm{a}}$, Merry-Jennifer Markham ${ }^{\mathrm{b}}$, Xiuli Liu ${ }^{\mathrm{a}, \mathrm{c}}$
}

\begin{abstract}
The link between immunosuppressive therapy and increased lymphoma risk is well established in patients with solid organ transplantation. Epstein-Barr virus-positive (EBV) diffuse large B-cell lymphoma (DLBCL) is known to be a complication in patients receiving methotrexate for rheumatoid arthritis, and the risk of lymphoma in inflammatory bowel disease (IBD) has raised concerns regarding the lymphoproliferative potential of immunomodulatory therapy. In this report, we describe a case of EBV-positive DLBCL arising within the colon of a patient affected by ulcerative pancolitis. The patient is a 73-year-old man with a history of IBD and polymyositis on long-term methotrexate therapy. Increasing age and long-term methotrexate therapy may simulate post-transplantation immunosuppression and contribute to lymphoma tumorigenesis in a segment of chronically inflamed colon.
\end{abstract}

Keywords: Epstein-Barr virus; B-cell lymphoma; Colon; Ulcerative pancolitis; Polymyositis; Methotrexate

\section{Introduction}

The link between immunosuppressive therapy and increased lymphoma risk is well established in patients who have undergone solid organ transplantation and in those with rheumatoid arthritis with or without methotrexate medication $[1,2]$. Of the non-Hodgkin lymphomas that develop in patients who have received a solid organ transplantation or who are on methotrexate for rheumatoid arthritis, up to $80 \%$ and $28 \%$, respectively, contain the Epstein-Barr virus (EBV) genome [3, 4]. It

Manuscript accepted for publication September 09, 2016

aDepartment of Pathology, Immunology and Laboratory Medicine, University of Florida, Gainesville, FL, USA

bivision of Hematology \& Oncology, College of Medicine, University of Florida, Gainesville, FL, USA

${ }^{c}$ Corresponding Author: Xiuli Liu, Department of Anatomic Pathology, Immunology and Laboratory Medicine, University of Florida, PO Box 100275, Gainesville, FL 32610, USA. Email: xiuliliu@ufl.edu

doi: http://dx.doi.org/10.14740/gr720e has been postulated that EBV-infected lymphocytes proliferate unchecked in the setting of impaired cell-mediated immunity, giving rise to lymphoma.

The increased risk of lymphoma in the setting of inflammatory bowel disease (IBD) has been associated with the use of immunomodulatory therapy such as azathioprine (AZA), 6-mercaptopurine (6-MP), and cyclosporine (CSA), and biologic therapies such as infliximab, a chimeric monoclonal antibody against human tumor necrosis factor alpha (TNF- $\alpha$ ), in medically refractory and fistulizing Crohn's disease and moderate to severe ulcerative colitis [5-10]. Most IBD-related lymphomas develop at sites of active intestinal inflammation in patients with longstanding disease. Less commonly seen are EBV-positive lymphomas arising within a pouch after proctocolectomy with ileal pouch anal anastomosis (IPAA) [11].

\section{Case Report}

Twenty-five years before being diagnosed with lymphoma, a 73-year-old man developed hematochezia and was diagnosed with ulcerative pancolitis. The patient was previously treated with a brief trial of prednisone, but was subsequently maintained on balsalazide at a dose of $6,750 \mathrm{mg}$ per day with good control of his symptoms, without diarrhea or rectal bleeding. Colonic biopsies 2 years ago showed histologic evidence of chronic colitis in his left colon and rectum. The patient was never treated with immune modulators or biologic agents for his ulcerative colitis. The patient also had longstanding polymyositis for the past 6 years, initially diagnosed on the basis of symptoms including proximal muscle weakness, a creatine kinase $(\mathrm{CK})$ level greater than 10,000 U/L (reference range: $44-196 \mathrm{U} / \mathrm{L}$ ), and a positive electromyography (EMG) study suggestive of an inflammatory myopathy. He had responded to high doses of steroids, and methotrexate was added several years ago with a gradual dose titration up to $20 \mathrm{mg}$ weekly. The patient's inflammatory muscle disease had never been completely controlled; serial CK levels remained in the range of $1,500-2,000 \mathrm{U} / \mathrm{L}$.

A few months prior to the current presentation, the patient developed dysphagia that was clinically felt to be a manifestation of polymyositis. The patient's blood pressure was $151 / 85$ $\mathrm{mm} \mathrm{Hg}$, his heart rate was 70 beats per minute, and his body weight was 203 pounds with a BMI of $32.3 \mathrm{~kg} / \mathrm{m}^{2}$. On physical 

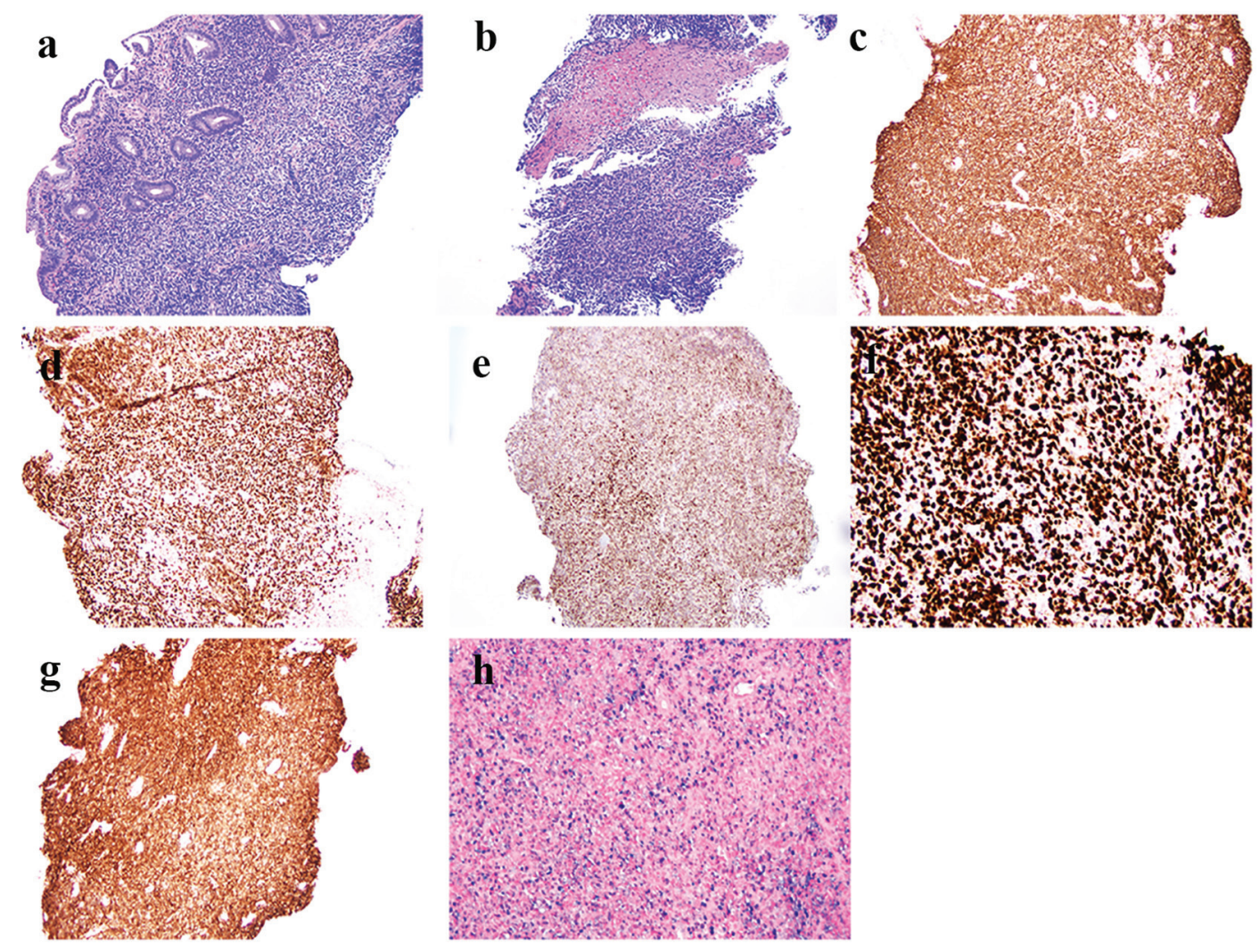

Figure 1. Histologic features and immunophenotype of diffuse large B-cell lymphoma in biopsy from the sigmoid colon polyp. (a) Atypical mononuclear infiltration in the lamina propria of the colonic biopsy (hematoxylin \& eosin stain, original magnification $x$ 100). (b) Focal tumor necrosis (hematoxylin \& eosin stain, original magnification $\times 100$ ). (c) Immunoreactivity of CD20 in tumor cells (immunoperoxidase stain, original magnification $\times 100$ ). (d) Immunoreactivity of PAX5 in tumor cells (immunoperoxidase stain, original magnification $\times 100)$. (e) Immunoreactivity of BCL6 in tumor cells (immunoperoxidase stain, original magnification $\times 100$ ). (f) High Ki67 labeling index (up to $80-90 \%$ ) (immunoperoxidase stain, original magnification $\times 200$ ). (g) Immunoreactivity of CD30 in tumor cells (immunoperoxidase stain, original magnification $\times 100)$. (h) Many Epstein-Barr virus-infected tumor cells by in situ hybridization (chromogenic in situ hybridization, original magnification $\times 200$ ).

examination, the patient had weakness in his lower extremities and some difficulty getting up out of a chair without the use of his hands. No lymphadenopathy was noted. Due to the suboptimal control of his polymyositis symptoms, mycophenolate mofetil (Cellcept) at a dose of $1,000 \mathrm{mg}$ per day was initiated and methotrexate was stopped on April 27, 2016, approximately 70 days prior to his surveillance colonoscopy in an outside hospital.

Esophagogastroduodenoscopy (EGD) performed for his dysphagia revealed abnormal motility of the esophagus, compatible with presbyesophagus, and a small hiatal hernia. A 17-mm Savary-type dilator was introduced for empirical dilatation. No definitive stricture was noted. A surveillance colonoscopy revealed normal mucosa in the cecum, ascending colon, and transverse colon. The descending colon and sigmoid colon, however, were characterized by erythema, friability, and congestion. In addition, a polypoid, nodular area with a slight stricture was noted in the sigmoid colon at $50 \mathrm{~cm}$. The rectum was endoscopically normal. Biopsies were taken from the descending colon and from the polypoid, nodular, strictured area in the sigmoid colon.

The sigmoid colon biopsy revealed a neoplasm comprised of a solid proliferation of large mononuclear cells with a high nucleus to cytoplasm (N/C) ratio, irregular nuclei, and distinct nucleoli (Fig. 1a). Tumor cell nuclei approximated the size of normal macrophage nuclei and were more than twice the size of normal lymphocytes. The tumor cells expanded the lamina propria and pushed the crypts apart, focally infiltrating the crypt epithelium (not shown). There was focal necrosis within the tumor (Fig. 1b). By immunohistochemistry, the tumor cells were diffusely positive for CD20 (Fig. 1c), PAX5 (Fig. 1d), and CD30 (Fig. 1g), strongly positive for BCL2 and MUM1 (not shown), and variably reactive for BCL6 (Fig. 1e). There was a high Ki67 labeling index (about 80-90\%) (Fig. 1f). Tumor cells were negative for CD3, CD5, CD10, cyclin D1, and SOX-11. The tumor cells were positive for EBV by in situ hybridization (Fig. 1h). A similar atypical lymphoid infiltrate was noted in the lamina propria (Fig. 2a) and crypt epithelium (Fig. $2 b$ ) in the descending colon biopsy. In addition, the descending colon biopsy showed mildly active chronic colitis (not shown). The overall histology and immunophenotype supported a diagnosis of an EBV-positive diffuse large B-cell lymphoma (DLBCL), non-germinal center type, involving the descending and sigmoid colon and arising in a background of ulcerative 

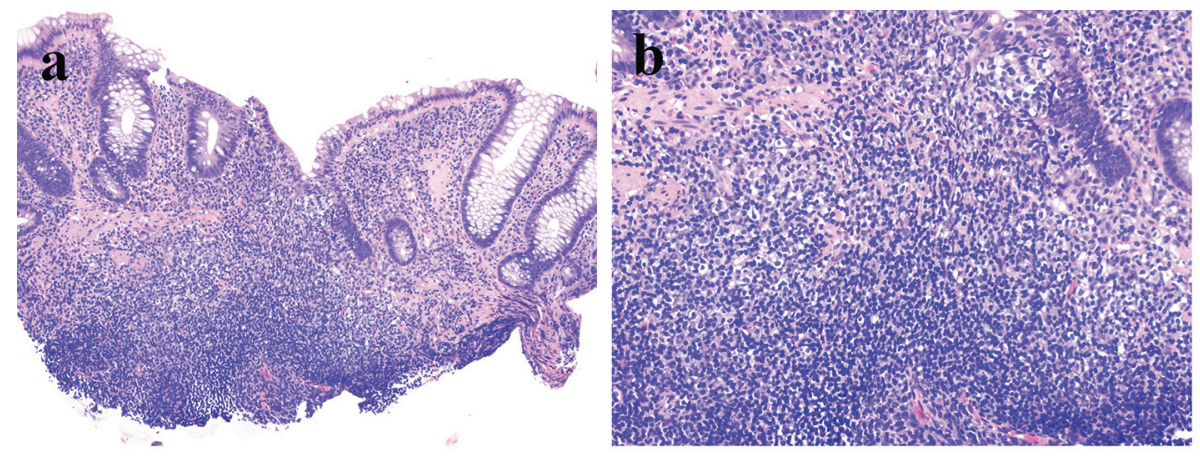

Figure 2. Atypical mononuclear cells are present in the lamina propria and focally infiltrate the crypt epithelium (a: hematoxylin \& eosin stain, original magnification $\times 100$; b: hematoxylin \& eosin stain, original magnification $\times 200$ ).

colitis.

Staging positron emission tomography with computed tomography (PET-CT) revealed intense focal pathologic uptake in the colon corresponding to the patient's biopsied lymphoma in a background of generalized physiologic uptake. There were no additional sites of increased uptake. The patient is currently scheduled to receive six cycles of dose-adjusted rituximab, etoposide, prednisolone, oncovin, cyclophosphamide, and hydroxydaunorubicin (DA-R-EPOCH) chemotherapy.

\section{Discussion}

The risk of lymphoma in IBD has been associated with the use of immunomodulatory therapy such as AZA, 6-MP, and CSA, and the use of biologics such as infliximab, in medically refractory and fistulizing Crohn's disease and moderate to severe ulcerative colitis [5, 11]. Cases of EBV-positive lymphoma arising within a pouch after proctocolectomy with IPAA have also been reported [11].

Here we report a case of EBV-positive DLBCL involving the colon in a patient with ulcerative colitis, whose IBD symptoms were well controlled with balsalazide. The patient also had a history of polymyositis, which had been treated with long-term methotrexate. The tumorigenesis of EBV-positive DLBCL in this case may be related to the patient's old age (73 years), the long-term use of methotrexate, and IBD. It is arguable whether this represents an EBV-positive DLBCL of the elderly or EBV-positive methotrexate-associated DLBCL. EBV-positive DLBCL of the elderly, initially described in 2003, is a provisional entity in the 2008 World Health Organization classification system and is defined as an EBV-positive monoclonal large B-cell proliferation that occurs in patients greater than 50 years of age and in whom there is no known immune deficiency, history of transplantation, or history of lymphoma [12-15].

Our patient had a history of polymyositis treated with long-term methotrexate, which was stopped 70 days prior to his colonoscopy. Long-term low-dose methotrexate therapy has been described in association with an EBV-positive, CD30-positive large B-cell lymphoma in one patient with a polymyositis-like condition [2]. The patient responded well to oral prednisone and acyclovir without cytotoxic chemothera- py. Our case most likely represents an EBV-positive DLBCL related to methotrexate use. The manifestation of lymphoma in the colon may be related to the patient's ulcerative colitis, as DLBCL has been reported to be associated with chronic inflammation [16]. Furthermore, most IBD-related lymphomas develop at sites of active intestinal inflammation in patients with longstanding disease [5-10].

In summary, we report a case of EBV-positive DLBCL involving the colon in a background of longstanding ulcerative colitis in a patient with polymyositis, treated with long-term methotrexate. Recognition of this entity is clinically significant as it may show spontaneous remission after cessation of methotrexate $[2,4]$. In cases without spontaneous remission, other therapeutic modalities, including cytotoxic chemotherapy, may be necessary.

\section{Disclosure}

None.

\section{Grant Support}

None.

\section{References}

1. Hoshida Y, Xu JX, Fujita S, Nakamichi I, Ikeda J, Tomita Y, Nakatsuka S, et al. Lymphoproliferative disorders in rheumatoid arthritis: clinicopathological analysis of 76 cases in relation to methotrexate medication. J Rheumatol. 2007;34(2):322-331.

2. Kamel OW, Weiss LM, van de Rijn M, Colby TV, Kingma DW, Jaffe ES. Hodgkin's disease and lymphoproliferations resembling Hodgkin's disease in patients receiving long-term low-dose methotrexate therapy. Am J Surg Pathol. 1996;20(10):1279-1287.

3. Reijasse D, Le Pendeven C, Cosnes J, Dehee A, Gendre JP, Nicolas JC, Beaugerie L. Epstein-Barr virus viral load in Crohn's disease: effect of immunosuppressive therapy. Inflamm Bowel Dis. 2004;10(2):85-90. 
4. Niitsu N, Okamoto M, Nakamine H, Hirano M. Clinicopathologic correlations of diffuse large B-cell lymphoma in rheumatoid arthritis patients treated with methotrexate. Cancer Sci. 2010;101(5):1309-1313.

5. Dayharsh GA, Loftus EV, Jr., Sandborn WJ, Tremaine WJ, Zinsmeister AR, Witzig TE, Macon WR, et al. Epstein-Barr virus-positive lymphoma in patients with inflammatory bowel disease treated with azathioprine or 6-mercaptopurine. Gastroenterology. 2002;122(1):72-77.

6. Calaminici MR, Sheaff MT, Norton AJ, Feakins RM. Ileocaecal Epstein-Barr virus-positive lymphoproliferative disorder complicating Crohn's disease. Histopathology. 1999;35(4):388-390.

7. Kumar S, Fend F, Quintanilla-Martinez L, Kingma DW, Sorbara L, Raffeld M, Banks PM, et al. Epstein-Barr virus-positive primary gastrointestinal Hodgkin's disease: association with inflammatory bowel disease and immunosuppression. Am J Surg Pathol. 2000;24(1):66-73.

8. Li S, Borowitz MJ. Primary Epstein-Barr virus-associated Hodgkin disease of the ileum complicating Crohn disease. Arch Pathol Lab Med. 2001;125(3):424-427.

9. Losco A, Gianelli U, Cassani B, Baldini L, Conte D, Basilisco G. Epstein-Barr virus-associated lymphoma in Crohn's disease. Inflamm Bowel Dis. 2004;10(4):425429.

10. Wong NA, Herbst H, Herrmann K, Kirchner T, Krajewski AS, Moorghen M, Niedobitek F, et al. EpsteinBarr virus infection in colorectal neoplasms associated with inflammatory bowel disease: detection of the virus in lymphomas but not in adenocarcinomas. J Pathol. 2003;201(2):312-318.

11. Schwartz LK, Kim MK, Coleman M, Lichtiger S, Chadburn A, Scherl E. Case report: lymphoma arising in an ileal pouch anal anastomosis after immunomodulatory therapy for inflammatory bowel disease. Clin Gastroenterol Hepatol. 2006;4(8):1030-1034.

12. Nakamura S, Jaffe ES, Swerdlow SH. eds. EBV positive diffuse large B-cell lymphoma of the elderly. In: Swerdlow SH, Campo E, Harris NL, et al., eds. WHO Classification of Tumors of Haematopoietic and Lymphoid Tissues. Lyon, France: International Agency for Research on Cancer; 2008.

13. Oyama T, Ichimura K, Suzuki R, Suzumiya J, Ohshima K, Yatabe Y, Yokoi T, et al. Senile EBV+ B-cell lymphoproliferative disorders: a clinicopathologic study of 22 patients. Am J Surg Pathol. 2003;27(1):16-26.

14. Ok CY, Papathomas TG, Medeiros LJ, Young KH. EBVpositive diffuse large B-cell lymphoma of the elderly. Blood. 2013;122(3):328-340.

15. Menon MP, Pittaluga S, Jaffe ES. The histological and biological spectrum of diffuse large B-cell lymphoma in the World Health Organization classification. Cancer J. 2012;18(5):411-420.

16. Boroumand N, Ly TL, Sonstein J, Medeiros LJ. Microscopic diffuse large B-cell lymphoma (DLBCL) occurring in pseudocysts: do these tumors belong to the category of DLBCL associated with chronic inflammation? Am J Surg Pathol. 2012;36(7):1074-1080. 